$56^{\text {ème }}$ Congrès de la SFMBCB, 03008 (2011)

DOI: $10.1051 / \mathrm{sfmbcb} / 20115603008$

(C) Owned by the authors, published by EDP Sciences, 2011

\title{
Contrôles qualités et imagerie odonto-stomatologique : aspects réglementaires et mise en ouvre pratique
}

\author{
Salmon $\mathbf{B}^{1}$, Ledenmat $\mathrm{D}^{2}$, Maman $\mathbf{L}^{1}$, Ejeil AL ${ }^{1}$ \\ ${ }^{1}$ Département de Médecine buccale et Chirurgie buccale, Faculté de Chirurgie dentaire, \\ Université Paris Descartes, Paris, France \\ ${ }^{2}$ Laboratoire de Radiologie Imagerie et Biophysique,Faculté de Chirurgie dentaire, Université \\ Paris Descartes, Paris, France \\ drbenjaminsalmon@hotmail.fr
}

L'Autorité de Sûreté Nucléaire (ASN) définit la radioprotection comme l' « ensemble des règles, des procédures et des moyens de prévention et de surveillance visant à empêcher ou à réduire les effets nocifs des rayonnements ionisants produits sur les personnes directement ou indirectement, y compris par les atteintes portées à l'environnement ». Contraignante, la nouvelle réglementation impose à la profession de prendre des mesures de radioprotection strictes et identiques à celles de tous les professionnels exposés aux rayonnements ionisants :

- Formation données aux professionnels de santé pour la radioprotection des patients,

- Désignation d'une personne compétente en radioprotection,

- Mise aux normes des installations avec un contrôle technique quinquennal,

- Mise en place des contrôles qualité internes, externe et audit.

Découlant du principe d'optimisation, les contrôles qualité consistent à vérifier le maintien des performances de la chaîne radiologique permettant l'obtention de clichés de qualité pour un minimum de rayonnements ionisants dans des conditions de sécurité optimales. La décision de l'Afssaps fixant les modalités du contrôle qualité des installations de radiologie dentaire a été publiée au Journal officiel le 26 décembre 2008. La mise en place progressive du contrôle qualité est prévue en fonction de la date de construction du générateur de rayons $\mathrm{X}$. On distingue :

1. Le contrôle qualité interne réalisé par le praticien (ou un prestataire) : il est initial, puis de périodicité trimestrielle pour les techniques numériques et mensuelle pour les techniques analogiques (argentiques),

2. Le contrôle qualité externe réalisé par un organisme de contrôle agréé : il est initial, puis de périodicité quinquennale [avant le 26/12/2009 pour les générateurs de plus de 10 ans (1998), avant le 26/12/2010 pour les générateurs âgés de 5 à 10 ans, avant le 26/12/2011 pour les générateurs de moins de 5 ans (2003)],

3. Un audit annuel du contrôle interne réalisé par un organisme agréé par l’Afssaps (avant le 26/12/2010 et avant 26/12/2012 pour une mise en service après le 26/12/2009).

L'ensemble des opérations de contrôle doit faire l'objet d'une traçabilité. Le praticien doit établir, tenir à jour et mettre à la disposition de l’organisme de contrôle de qualité externe, l'inventaire et le registre des opérations de maintenance et de contrôle de qualité.

La mise en œuvre pratique du contrôle qualité interne vise à exposer des «objets test » distincts selon les modalités intra ou extra-orales et selon les dispositifs numériques ou argentiques. Les images obtenues doivent valider une résolution spatiale et une résolution à bas contraste acceptables (par exemple, au moins 6,3 pl. $\mathrm{mm}^{-1}$ en imagerie intra-orale numérique et 2,5 pl. $\mathrm{mm}^{-1}$ pour une radiographie panoramique). Les fantômes qualité dédiés à l'imagerie tridimensionnelle (Cone Beam Computed Tomography) sont en cours de conception et d'évaluation au laboratoire. Les critères de qualité des images seront spécifiques et plus complexes (précision dimensionnelle, restitution des formes selon les plans de coupe, rapport signal/bruit, artéfacts...). 